\title{
Hospital admissions for alcohol-related problems in concomitance with weekends, New Year's Eve and Christmas Eve: Myth or reality?
}

\author{
Giuseppe Lippi, ${ }^{1,2}$ Anna Ferrari, ${ }^{2}$ Chiara Bovo, ${ }^{3}$ Gianfranco Cervellin ${ }^{4}$ \\ ${ }^{1}$ Section of Clinical Biochemistry, University Hospital of Verona; ${ }^{2}$ Service of Laboratory Medicine, \\ University Hospital of Verona; ${ }^{3}$ Medical Direction, University Hospital of Verona; ${ }^{4}$ Emergency Department, \\ University Hospital of Parma, Italy
}

\begin{abstract}
This retrospective observational study was carried out by searching the database of the laboratory information system for identifying all requests for alcohol testing placed from emergency departments and intensive care units of the University Hospital of Verona between June 29th, 2012 and December 31st, 2018. The study population consisted of 7488 patients. The number of alcohol tests was more than double in concomitance with New Year's Eve than in the rest of the year $(7.6 \pm 6.1$ vs $3.1 \pm 2.8$ requests/day; $\mathrm{P}<0.001)$, whereas blood alcohol concentration was similar $(1.55$ vs $1.12 \mathrm{~g} / \mathrm{L} ; \mathrm{P}=0.308)$. The risk of measuring alcohol concentrations $>0.1 \mathrm{~g} / \mathrm{L}$ and $>1.0 \mathrm{~g} / \mathrm{L}$ was 1.9 -fold and 1.6-fold higher in concomitance with New Year's Eve. In multivariate analysis, younger age, female sex and alcohol testing during New Year's Eve remained significant predictors of alcohol concentrations $>0.1$ $\mathrm{g} / \mathrm{L}$ and $>1.0 \mathrm{~g} / \mathrm{L}$. The requests for alcohol testing were similar in concomitance with Christmas Eve and in other periods of the year, whilst number of requests $(4.0 \pm 3.2 v s \quad 2.8 \pm 2.5$ requests/day; $\mathrm{P}<0.001)$ and concentration ( 1.37 vs $0.77 \mathrm{~g} / \mathrm{L} ; \mathrm{P}<0.001)$ were higher during weekends than in other weekdays.
\end{abstract}

\footnotetext{
Correspondence: Giuseppe Lippi, Section of Clinical Biochemistry, University Hospital of Verona, Piazzale L.A. Scuro, 37134 Verona, Italy.

E-mail: giuseppe.lippi@univr.it

Key words: Alcohol; Intoxication; Epidemiology; Emergency medicine.

Contributions: the authors contributed equally

Conflict of interest: the authors declare no potential conflict of interest.

Funding: none.

Ethical statement: due to the retrospective nature of the study, and to the anonymous way in which the data was managed, informed consent was not required.

Received for publication: 3 January 2019.

Revision received: 28 January 2019

Accepted for publication: 29 January 2019

This work is licensed under a Creative Commons Attribution 4.0 License (by-nc 4.0)

(C) Copyright: the Author(s), 2019

Licensee PAGEPress, Italy

Emergency Care Journal 2019; 15:8024

doi:10.4081/ecj.2019.8024
}

\section{Introduction}

Alcohol abuse is associated with a high burden of morbidity and mortality in the general population, paralleled by an increasing pattern of alcohol consumption in several Countries around the world. ${ }^{1}$ It is widely perceived, and somewhat demonstrated, that some social events are associated with heavier alcohol consumption and, consequently, with a higher number of alcohol-related problems. $^{2-5}$

Data collection on hospital admission for alcohol-related problems is challenging, mainly for difficulties in collecting history on prior-day or prior-week drinking, ${ }^{6}$ but also because the different aspects of clinical assessment do not efficiently contribute to reliably mirror the blood alcohol concentration. ${ }^{7}$ Moreover, the lack of routine measurement of alcohol concentration may further contribute to amplify the epidemiological bias, since this type of laboratory testing is sometimes prescribed when the clinical picture is unclear, or when mandated by legal authorities. ${ }^{8}$

With the aforementioned limitations, the drinking patterns of some social communities, such as college students, suggests that heavy drinking may be particularly frequent in concomitance with special events and holidays (New Year's Eve or birthdays, among others). ${ }^{4}$ Although recent data seems to confirm that alcohol consumption may be highly event-specific, thus suggesting to plan targeted intervention strategies aimed at reducing or preventing alcohol-related harmful events on a population level, ${ }^{9}$ other studies failed to show similar temporal patterns, except for weekend peaks. ${ }^{10}$ Therefore, this study was aimed to analyze the temporal trends of alcohol tests and relative results performed in patients admitted to a large University Hospital in Northern Italy.

\section{Materials and Methods}

This retrospective observational study was carried out with an electronic search in the database of the local laboratory information system (LIS) aimed at identifying all requests for alcohol testing placed from emergency departments (EDs) or intensive care units (ICUs) of the University Hospital of Verona, between June 29th, 2012 (i.e., the last searchable date in the database) and December 31st, 2018. The University Hospital of Verona comprises two large academic centers (Borgo Roma and Borgo Trento Hospitals), averaging $\sim 1500$ beds and serving an area with a population of approximately 400,000 inhabitants. Each hospital has ED and ICU; the facility is also a reference Trauma Center. Laboratory tests requests are always placed using a computerized physician order entry (Metafora Informatica Srl, Milan, Italy) and results are permanently stored in the LIS. Alcohol concentration is conventionally measured in lithium-heparin plasma, with an alcohol dehydrogenase assay, on Siemens Dimension Vista (Siemens 
Healthcare Diagnostics, Camberley, UK). The methods were not changed throughout the study period. The measuring range of this method is comprised between $0.1-3.0 \mathrm{~g} / \mathrm{L}$; concentrations $>3.0 \mathrm{~g} / \mathrm{L}$ are automatically diluted 1:4 with sample buffer and then retested to obtain a precise value. The imprecision of this assay is comprised between $1.5-2.5 \%$, as declared by the manufacturer. The local laboratory is accredited according to the ISO 15189 standards and the quality of test results was validated throughout the study period by regular performance of internal quality controls and participation to a certified external quality assessment scheme. Alcohol testing requests and relative results were divided between those performed in concomitance with specific periods of the year (i.e., New Year's Eve: December 31st and January 1st; Christmas Eve: December 24th and 25th; weekends: Saturdays and Sundays) and those carried out in the remaining periods. Continuous data were shown as median value and interquartile range (IQR), whilst categorical data were presented as number and percentage. Significance of differences was assessed with Mann-Whitney and Chi square tests for continuous and categorical data, respectively. The statistical analysis was carried out with Analyse-it (Analyse-it Software Ltd, Leeds, UK) and MedCalc version 12.3.0 (MedCalc Software, Mariakerke, Belgium). The statistical significance was set at $\mathrm{P}<0.05$.

\section{Results}

The final study population consisted of 7488 patients who had a request for alcohol testing placed from the local EDs $(n=7457$; $99.6 \%)$ or ICUs $(n=31 ; 0.4 \%)$ throughout the study period $(2379$ days). Albeit the number of alcohol tests was more than double in concomitance with New Year's Eve than in the remaining period of the year ( $7.6 \pm 6.1$ vs $3.1 \pm 2.8$ requests per day; $\mathrm{P}<0.001)$, the blood alcohol concentration measured in these two days (median, 1.55 $\mathrm{g} / \mathrm{L}$; IQR, 0.25-2.16 g/L) was slightly but not significantly higher than in the remaining period of the year (median, $1.12 \mathrm{~g} / \mathrm{L}$; IQR, 0.10-2.31 g/L; $\mathrm{P}=0.308$ ). Patients who had a request for alcohol testing placed in concomitance with New Year's Eve were younger (32 vs 45 years; $\mathrm{P}<0.001$ ) and were slightly more frequently women ( 32 vs $24 \%$; $\mathrm{P}=0.045$ ) than in the rest of the year (Table 1).

The number of patients with measurable alcohol concentration (i.e., $>0.1 \mathrm{~g} / \mathrm{L}$ ) was 63/84 (75\%) in concomitance with New Year's Eve compared to $4560 / 7404$ in the remaining period of the year $(62 \% ; \mathrm{P}=0.001)$, whilst that of patients with toxic alcohol concentration (i.e., $>1 \mathrm{~g} / \mathrm{L})^{11}$ was $53 / 84(63 \%)$ in concomitance with New Year's Eve compared to 3814/7404 in the remaining period of the year ( $52 \% ; \mathrm{P}=0.023)$. Overall, the risk of measuring alcohol concentrations $>0.1 \mathrm{~g} / \mathrm{L}$ and $>1.0 \mathrm{~g} / \mathrm{L}$ was 1.9 -fold (odds ratio [OR], $1.87 ; 95 \%$ confidence interval [95\% CI], 1.14-3.07) and 1.6- fold (OR, 1.61; 95\% CI, 1.03-2.51) higher in concomitance with New Year's Eve than in the rest of the year, respectively (Figure 1).

In multivariate linear regression analysis, alcohol concentration was found to be significantly associated with younger age (beta coefficient, $-0.01 ; \mathrm{P}<0.001$ ), female sex (beta coefficient, $0.174 ; \mathrm{P}<0.001$ ), but not with alcohol testing concomitant with New Year's Eve (beta coefficient, 0.05; $\mathrm{P}=0.970$ ). Unlike these findings, younger age (beta coefficient, 0.04; $\mathrm{P}<0.001$ ), female sex (beta coefficient $0.04 ; \mathrm{P}=0.002$ ) and alcohol testing concomitant with New Year's Eve (beta coefficient, 0.104; $\mathrm{P}=0.019$ ) remained significant predictors of alcohol concentration $>0.1 \mathrm{~g} / \mathrm{L}$, whilst younger age (beta coefficient, 0.04; $\mathrm{P}<0.001$ ), female sex (beta coefficient $0.04 ; \mathrm{P}=0.003$ ) and alcohol testing concomitant with New Year's Eve (beta coefficient, 0.085; $\mathrm{P}=0.049$ ) remained also significant predictors of alcohol concentration $>1 \mathrm{~g} / \mathrm{L}$. Albeit the requests for alcohol testing were slightly higher in concomitance with Christmas Eve than in other periods of the year, this differ-

Table 1. Characteristics of patients with alcohol test requests placed in concomitance with New Year's Eve and in the remaining period of the year.

\begin{tabular}{lccc} 
Variables & New Year's Eve & Rest of the year & P \\
Number of requests & 84 in 11 days (7.6/day) & 7404 in 2368 days (3.1/day) & $<0.001$ \\
Age (years) & $32(\mathrm{IQR}, 21-51)$ & $45(\mathrm{IQR}, 31-59)$ & $<0.001$ \\
\hline Sex (women) & $27 / 84(32 \%)$ & $1749 / 7404(24 \%)$ & 0.045 \\
Alcohol & & & \\
$\quad$ Median concentration (g/L) & $1.55(\mathrm{IQR}, 0.25-2.16)$ & $1.12(\mathrm{IQR}, 0.10-2.31)$ & 0.308 \\
$\quad$ Patients with $>0.1 \mathrm{~g} / \mathrm{L}$ & $63 / 84(75 \%)$ & $4560 / 7404(62 \%)$ & 0.001 \\
$\quad$ Patients with $>1.0 \mathrm{~g} / \mathrm{L}$ & $53 / 84(63 \%)$ & $3814 / 7404(52 \%)$ & 0.023 \\
\hline
\end{tabular}

IQR, interquartile range.

Table 2. Characteristics of patients with alcohol test requests placed in concomitance with new weekends and in the remaining days of the week.

\begin{tabular}{lccc} 
Variables & Weekends & Weekdays & P \\
Number of requests & 2721 in 680 days (4.0/day) & 4767 in 1699 days (2.8/day) & $<0.001$ \\
Age (years) & $42(\mathrm{IQR}, 27-59)$ & $46(\mathrm{IQR}, 33-60)$ & $<0.001$ \\
Sex (women) & $632 / 2721(23 \%)$ & $1174 / 4767(25 \%)$ & 0.382 \\
Alcohol & & & $<0.001$. \\
$\quad$ Median concentration (g/L) & $1.37(\mathrm{IQR}, 0.10-2.35)$ & $0.77(\mathrm{IQR}, 0.10-2.24)$ & $<0.001$ \\
$\quad$ Patients with $>0.1 \mathrm{~g} / \mathrm{L}$ & $1819 / 2721(67 \%)$ & $2836 / 4767(59 \%)$ & $<0.001$ \\
$\quad$ Patients with $>1.0 \mathrm{~g} / \mathrm{L}$ & $1541 / 2721(57 \%)$ & $2339 / 4767(49 \%)$ & \\
\hline
\end{tabular}

$\mathrm{IQR}$, interquartile range. 
ence did not reach statistical significance $(3.9 \pm 2.8$ vs $3.2 \pm 2.9$ requests per day; $\mathrm{P}=0.077$ ), nor were significantly different the alcohol concentration $(2.18$ vs $2.00 \mathrm{~g} / \mathrm{L} ; \mathrm{P}=0.417)$ and the number of patients with either measurable or toxic alcohol concentrations (Figure 1).

Interestingly, a sub-analysis of data revealed that the number of alcohol tests was also higher during weekends than in the other weekdays ( $4.0 \pm 3.2$ vs $2.8 \pm 2.5$ requests per day; $\mathrm{P}<0.001$ ), and that the blood alcohol concentration measured in these two days (median, $1.37 \mathrm{~g} / \mathrm{L}$; IQR, $0.10-2.35 \mathrm{~g} / \mathrm{L}$ ) was nearly double than in the remaining period of the week (median, $0.77 \mathrm{~g} / \mathrm{L}$; IQR, 0.10-2.24 $\mathrm{g} / \mathrm{L} ; \mathrm{P}<0.001$ ) (Table 2). Similarly, the number of patients with measurable alcohol concentration (i.e., $>0.1 \mathrm{~g} / \mathrm{L}$ ) measured during the weekends was significantly higher compared to the remaining weekdays (67\% vs 59\%; OR, 1.37 and 95\% CI, 1.24-1.51; $\mathrm{P}<0.001)$. The number of patients with toxic alcohol concentration (i.e., $>1 \mathrm{~g} / \mathrm{L}$ ) measured during the weekends was also significantly higher compared to the other weekdays (57\% vs 49\%; OR, 1.45 and 95\% CI, 1.23-1.49; $\mathrm{P}<0.001$ ) (Figure 1).

\section{Discussion and Conclusions}

Although it has been hypothesized that some social events may considerably increase alcohol consumption and alcohol-related complications in many worldwide countries, the literature data are somehow controversial. Poikolainen et al. studied fatal alcohol intoxications in Finland, ${ }^{12}$ and found that fatal alcohol poisonings were especially frequent in midsummer and Christmas compared to all other periods of the year. In a subsequent investigation Mäkelä et al. also studied the temporal variation in deaths attributable to alcohol intoxication in the Finnish death register and the Finnish Drinking Habits Survey. ${ }^{3}$ In close agreement with our findings, the risk of intoxication-related deaths during the New Year's day increased by $48 \%$ (OR, 1.48 95\% CI, 1.20-1.80) in men and by $77 \%$ (OR, 1.77; 95\% CI, 1.08-2.74) in women, respectively. Intoxication-related deaths were also found to be between 1.6-fold and 2.2-fold higher on Saturdays, Sundays and in concomitance with Christmas Eve. In a following study based on telephone surveys of Canadian residents drinking alcohol at least once per month, Kushnir and Cunningham also found a consistent temporal trend in alcohol consumption, with higher peaks on Fridays, Saturdays, Christmas and New Year's Eve. ${ }^{9}$ Foster et al. explored the trend of alcohol consumption and alcohol-related road accidents in young Swiss men, ${ }^{13}$ observing that the overall volume of drinking and alcohol-related road accidents were up to 4-fold higher during New Year's Eve and in the period between Friday evening and Saturday morning, whilst a minor peak was noticed during Christmas Eve. More recently Comelli et al. reviewed the data of all patients admitted to a large urban Italian ED for alcoholrelated problems, ${ }^{10}$ and found that the largest number of visits was recorded during the weekends, whilst no clear seasonality could be observed.

The results of our retrospective analysis of alcohol tests performed in a large Italian University Hospital pave the way to formulate some interesting considerations. Overall, the number of requests for alcohol testing placed from the local EDs or ICUs in concomitance with New Year's Eve was more than double than in the remaining period of the year, and this figure is then mirrored by a similar risk of measuring alcohol concentrations $>0.1 \mathrm{~g} / \mathrm{L}$ and $>1.0 \mathrm{~g} / \mathrm{L}$ (Figure 1). Similar results were found for alcohol tests requested during weekends compared to other weekdays. On the other hand, the median alcohol concentration measured in concomitance with New Year's Eve was found to be slightly, but not significantly, higher than in the rest of the year, whilst that measured on weekends was significantly higher than in the rest of week. This would actually mean that the number of people admitted to the hospital for suspected alcohol intoxication is higher in concomitance with New Year's Eve, but the overall volume of alcohol drank of people admitted to the hospital is likely the same. Unlike these findings, both the number of patients and alcohol concentration measured during weekends were found to be considerably higher than in the remaining period of the week, whilst neither the number of test or the relative alcohol concentration was found to be significantly different in concomitance with the Christmas Eve.

This investigation has some clear limitations, mostly attributable to the study design. Basically, our analysis was based on data retrieved from the local LIS, so that no information was available on drinking patters, reasons underneath alcohol requests and clinical picture. Nevertheless, our results may still be of some importance for both establishing targeted interventions aimed at reducing or preventing the alcohol-related emergencies in certain periods of the year such as the New Year's Eve and weekends. On the other hand, they also provide valuable information for proactively addressing potential organizational issues in EDs and ICUs (i.e., overcrowding), as well as in clinical laboratories (availability of staff and reagents), which may emerge from larger hospital admission of critical patients during certain periods of the year.
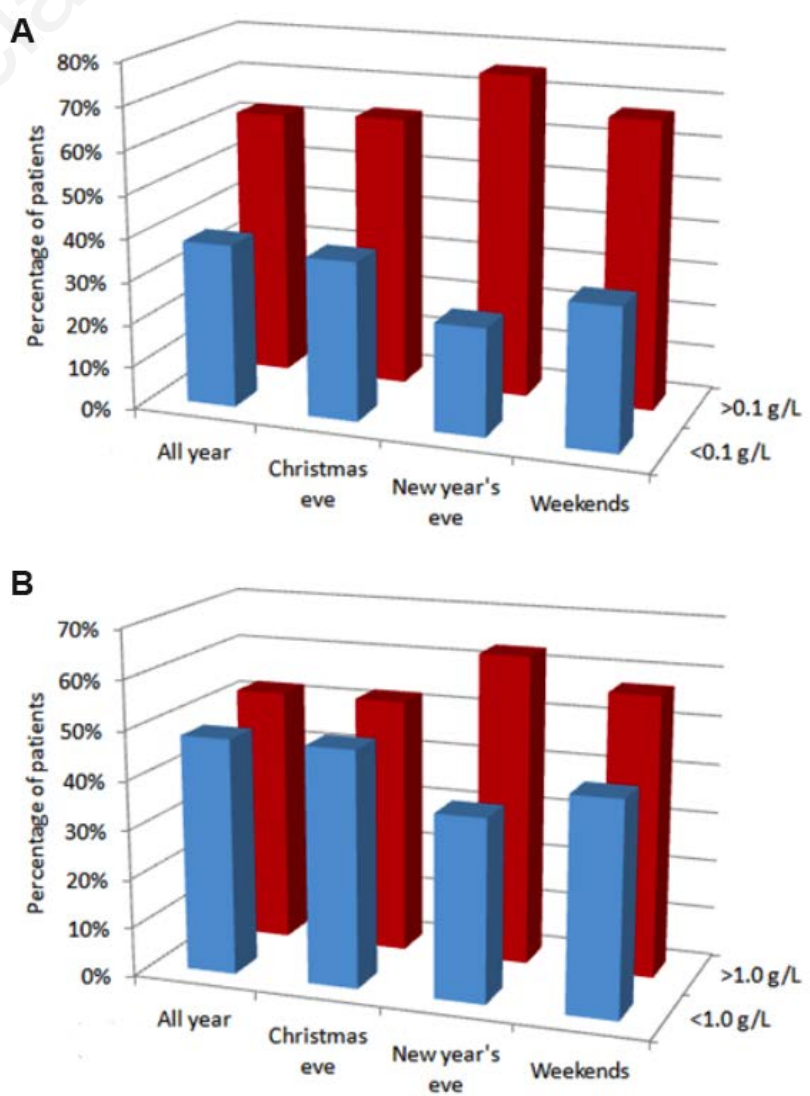

Figure 1. Percentage of patients admitted to the hospital with A) measurable $(>0.1 \mathrm{~g} / \mathrm{L})$ or $\mathrm{B})$ toxic $(>1.0 \mathrm{~g} / \mathrm{L})$ alcohol concentration. 


\section{References}

1. World Health Organization (WHO). Global status report on alcohol and health. Geneva, Switzerland: World Health Organization; 2014.

2. Del Boca FK, Darkes J, Greenbaum PE, et al. Up close and personal: temporal variability in the drinking of individual college students during their first year. J Consult Clin Psychol 2004; 72:155-64.

3. Mäkelä P, Martikainen P, Nihtilä E. Temporal variation in deaths related to alcohol intoxication and drinking. Int $\mathrm{J}$ Epidemiol 2005;34:765-71.

4. Neighbors C, Atkins DC, Lewis MA, et al. Event-specific drinking among college students. Psychol Addict Behav 2011;25:702-7.

5. Neighbors C, Spieker CJ, Oster-Aaland L, et al. Celebration intoxication: an evaluation of 21 st birthday alcohol consumption. J Am Coll Health 2005;54:76-80.

6. Cherpitel CJ, Ye Y, Stockwell T, et al. Recall bias across 7 days in self-reported alcohol consumption prior to injury among emergency department patients. Drug Alcohol Rev 2018;37:382-8.
7. Olson KN, Smith SW, Kloss JS, et al. Relationship between blood alcohol concentration and observable symptoms of intoxication in patients presenting to an emergency department. Alcohol Alcohol 2013;48:386-9.

8. Li G, Keyl PM, Rothman R, et al. Epidemiology of alcoholrelated emergency department visits. Acad Emerg Med 1998;5:788-95.

9. Kushnir V, Cunningham JA. Event-specific drinking in the general population. J Stud Alcohol Drugs 2014;75:968-72.

10. Comelli I, Lippi G, Sanchis-Gomar F, et al. Visits for alcoholrelated problems in a large urban emergency department. Results of a 15-year survey. Acta Biomed 2018;88:514-8.

11. Vonghia L, Leggio L, Ferrulli A, et al. Acute alcohol intoxication. Eur J Intern Med 2008;19:561-7.

12. Poikolainen K, Leppänen K, Vuori E. Alcohol sales and fatal alcohol poisonings: a time-series analysis. Addiction 2002;97:1037-40.

13. Foster S, Gmel G, Estévez N, et al. Temporal patterns of alcohol consumption and alcohol-related road accidents in young Swiss men: seasonal, weekday and public holiday effects. Alcohol Alcohol 2015;50:565-72. 